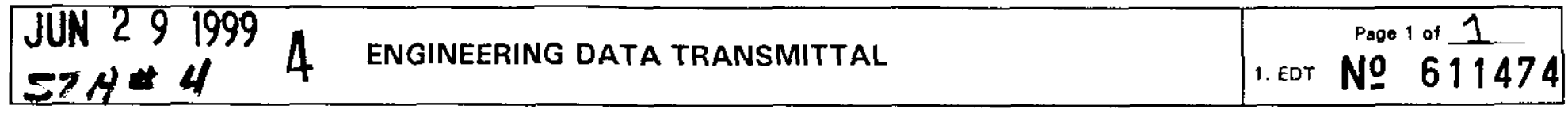

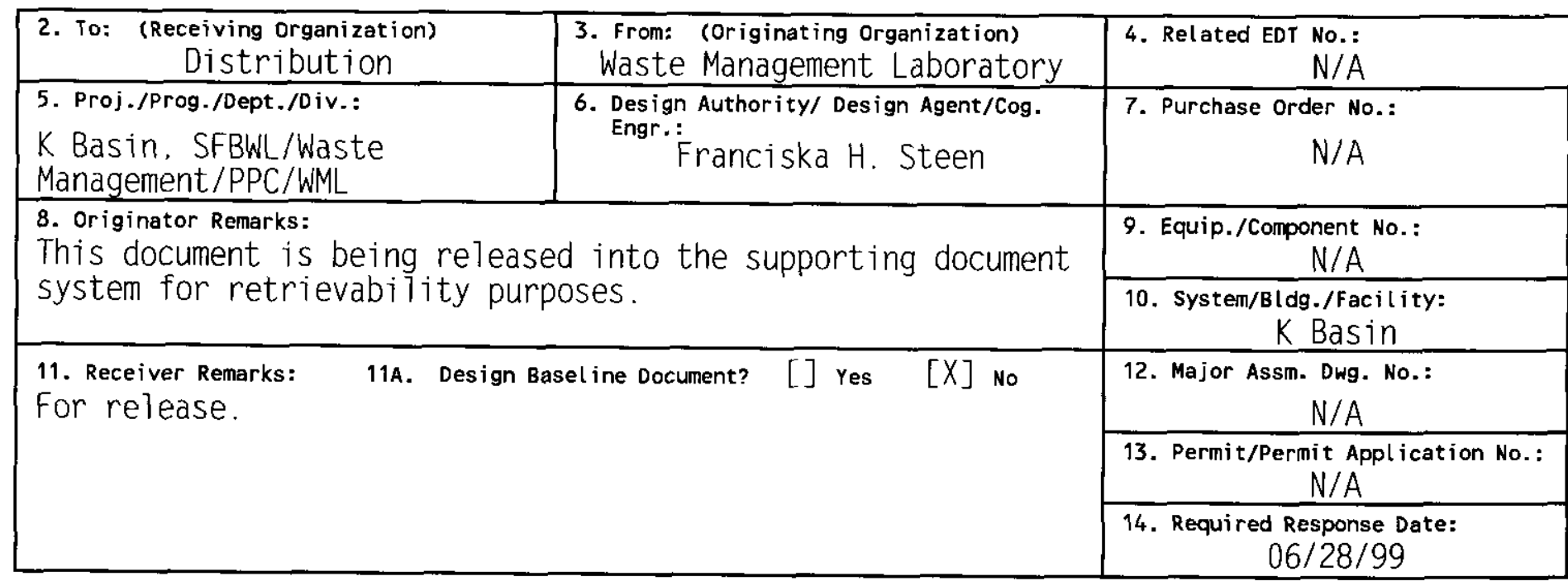

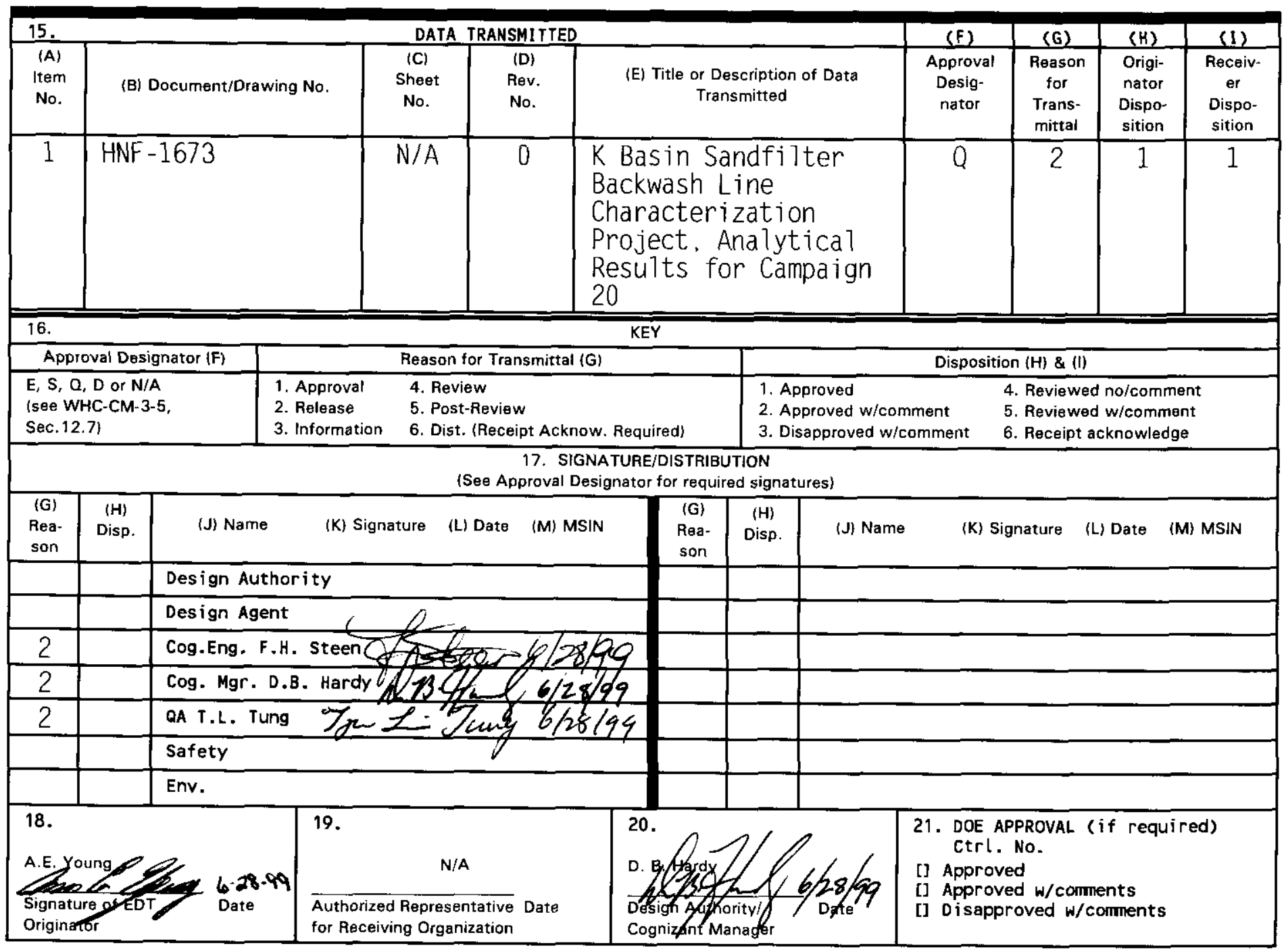




\section{K Basin Sandfilter Backwash Line Characterization Project, Analytical Results for Campaign 20}

Franciska H. Steen

Waste Management of Hanford, Inc., Richland, WA 99352

U.S. Department of Energy Contract DE-AC06-96RL13200

EDT/ECN: EDT-611474 UC: 2070

Org Code: $31 \mathrm{~B} 00$

Charge Code: LC087

B\&R Code: EW 3120074 Tota1 Pages: 15

Key Words: K Basin Sandfilter Backwash. Sandfilter, SBWL, Characterization, Campaign 20, K Basin

Abstract: N/A

TRADEMARK DISCLAIMER. Reference herein to any specific commercial product, process, or service by trade name, trademark, manufacturer, or otherwise, does not necessarily constitute or imply its endorsement, recommendation, or favoring by the United States Government or any agency thereof or its contractors or subcontractors.

Printed in the United States of America. To obtain copies of this document, contact: Document Control Services, P.O. Box 950, Mailstop H6-08, Richland HA 99352, Phone (509) 372-2420; Fax (509) 376-4989.
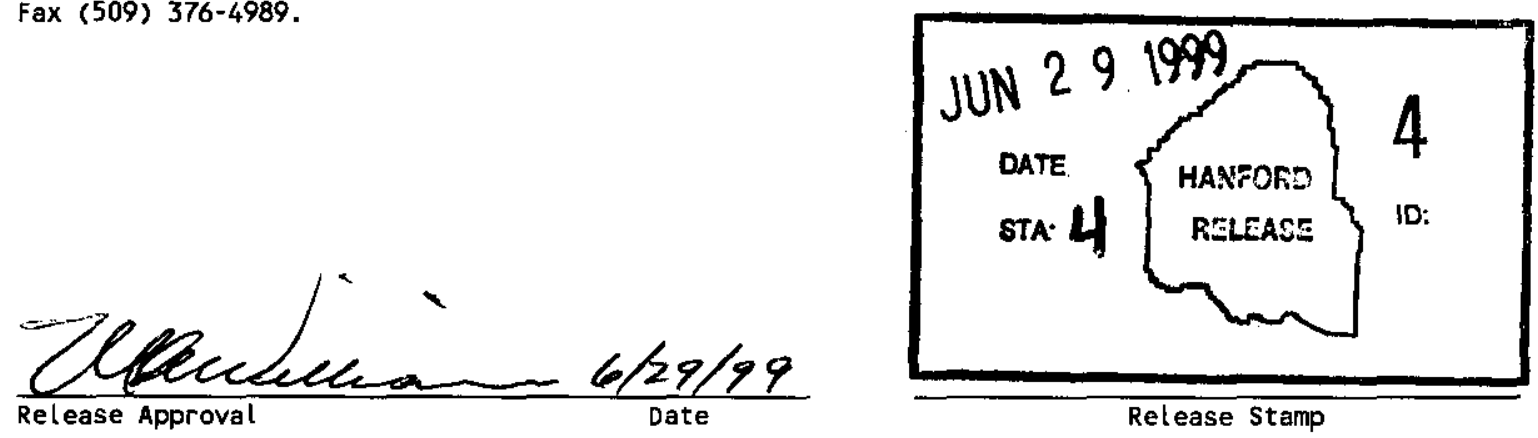

Approved for Public Release 
HNF-1673 REV. 0

\section{WASTE MANAGEMENT LABORATORY}

\section{$K$ BASIN SANDFILTER BACKWASH LINE CHARACTERIZATION PROJECT, ANALTYICAL RESULTS FOR CAMPAIGN 20}

Project Coordinator: Franciska H. Steen

Prepared for the U.S. Department of Energy

Office of Environmental Restoration and Waste Management

by

222-S Laboratory

Waste Management of Hanford, Inc. P.0. Box 700

Richl and, Washington 
HNF-1673, REV. 0

\section{WASTE MANAGEMENT LABORATORY}

\section{K BASIN SANDFILTER BACKWASH LINE CHARACTERIZATION PROJECT, ANALYTICAL RESULTS FOR CAMPAIGN 20}

\section{$\underline{\text { Summary }}$}

Sample 112KWBMF was taken from the K West Sandfilter Backwash Pit on June 1, 1999, and received by 222-S Laboratory on June 2, 1999. Analyses were performed on sample $112 \mathrm{KWBMF}$ in accordance with Letter of Instruction for K Basins Sandfilter Backwash Line Samples (LOI) in support of the K Basin Sandfilter Backwash Line Characterization Project.

\section{Appearance and Sample Handling}

Sample 112KWBMF consisted of approximately 280 milliliters of clear liquid with a small amount of black solids on the bottom of the container. Table 1 summarizes appearance information and over-the-top (OTR) dose readings performed on the sample.

Table 1. Appearance and OTR for K W Basin Sandfilter Backwash Sample

\begin{tabular}{|c|c|c|c|c|c|c|}
\hline $\begin{array}{c}\text { Customer } \\
\text { ID }\end{array}$ & $\begin{array}{c}\text { Lab } \\
\text { ID }\end{array}$ & Color & Clarity & $\begin{array}{c}\text { Organic } \\
\text { Layer }\end{array}$ & Solids & $\begin{array}{c}\text { OTR } \\
\text { (MRAD/HR) }\end{array}$ \\
\hline $112 \mathrm{KWBMF}$ & s99K000019 & None & Clear & None & Black & $<0.5$ \\
\hline
\end{tabular}

\section{Sample Preparation}

There were no anomalies observed nor exceptions to the data precision and accuracy requirements stated in LOI for the digestion of the sample. The K Basin Sample Digestion Data Sheet (Table 2) compiles analytical results associated with the digestion process. Determination of Data Correction Digestion/Dilution Factors (Table 3) presents the data used to determine the digestion/dilution conversion factor and the final calculated value.

Attachment 1 (Sample Processing Scheme for K West Basin Sandfilter Backwash Line Sample $112 \mathrm{KWBMF}$ ) illustrates the digestion process. Furthermore, this reference relates $\mathrm{K}$ West Basin identification numbers to their corresponding 222-S Laboratory Information Management System sample numbers. 


\section{HNF-1673, REV. 0}

Sample 112KWBMF was entered into the 222-S Laboratory Information Management System (LABCORE) as a solid sample. In order to satisfy the LABCORE upload of the raw data, a digest factor of $1 \mathrm{~g} / \mathrm{L}$ was used to convert the units for the results from $\mathrm{uCi} / \mathrm{L}$ to $\mathrm{uCi} / \mathrm{g}$. Therefore, the $\mathrm{uCi} / \mathrm{g}$ data results are equal to the $\mathrm{uCi} / \mathrm{L}$ data results. The $\mathrm{uCi} / \mathrm{L}$ results were converted to $\mathrm{uCi} / \mathrm{mL}$ on Tables $4 \mathrm{~A}, 4 \mathrm{~B}, 4 \mathrm{C}, 5,6$ and 7 . The volume and weight correction factors were also applied in order to correct the data results to the original sample.

\section{Analytical Results}

The data summary tables included in this report compile analytical results associated with each sample. Results are reported as activity of the digestate (S99K000019). These results were corrected for volume and weight of the entire sample. Analytical results for the digestate are presented in Tables 4A, 4B and 4C. Analytical results corrected to the original sample volume are presented in Tables 5,6 and 7. These tables present an estimated quantification limit (EQL) for each analysis. An EQL is equivalent to a practical quantification limit (as required by $\mathrm{LOI}$ ) and is calculated as the method detection limit multiplied by any dilution factor and by a factor of ten.

\section{Plutonium (238 Pu and 239/240Pu)}

There were no anomalies observed during sample analysis. The detection limit was much less than the customer specified detection limit of $4.2 \mu \mathrm{Ci} / \mathrm{L}$. The preparation blank did not show contamination. Standard recovery, precision and accuracy were all within acceptable limits.

\section{Uranium (U)}

There were no anomalies observed during sample analysis. The detection limit was much less than the customer specified detection limit of $23 \mathrm{mg} / \mathrm{L}$. The preparation blank did not show contamination. Standard recovery, precision, and accuracy were all within acceptable limits. 
HNF-1673, REV. 0

\section{Procedures}

Table 8 lists the analytical procedures used for performing sample analyses.

Table 8. Analytical Procedures

\begin{tabular}{|c|c|c|}
\hline Analysis & $\begin{array}{c}\text { Preparation } \\
\text { Procedure }\end{array}$ & $\begin{array}{c}\text { Analysis } \\
\text { Procedure }\end{array}$ \\
\hline $\begin{array}{c}\text { Pu 238 and } \\
\text { Pu239/240 }\end{array}$ & LA-505-162 Rev. C-0 & LA-953-104 Rev. B-0 \\
\hline $\mathrm{U}$ & LA-505-162 Rev. C-0 & LA-925-009 Rev. B-0 \\
\hline
\end{tabular}

\section{References}

DeFigh-Price, C., October 1996, Letter of Instruction for K Basins Sandfilter Backwash Line Samples, Revision 3, Internal Memorandum 2A100-96.018.

Markel, L.P., 1999, 222-S Laboratory Quality Assurance Plan, HNF-SD-CP-QAPP-016, Rev. 3C, Waste Management Federal Services of Hanford, Inc., Richland, WA 99352. 
Table 2. Sample Digestion Data Sheet (LA-505-162, Revision C-0)

\begin{tabular}{|c|c|c|c|c|}
\hline Step & $\begin{array}{c}\text { Labcore } \\
\text { Name }\end{array}$ & Parameter Deocription & Unit: & Datum \\
\hline 9.1 .2 .1 & & Labcore Samplo Number & $n / \bullet$ & s99k000019 \\
\hline 9.1.2.2 & ESTVOLO1 & Estimated totel volume of sample & $\mathbf{m L}$ & 275.8 \\
\hline 9.1 .2 .3 & DOSE-02 & Dose rate whindow closed & $\mathrm{mR} / \mathrm{hr}$ & $<0.5$ \\
\hline 9.1 .2 .3 & WODOSEO2 & Dose rate window open & $\mathrm{mrad} / \mathrm{hr}$ & $<0.5$ \\
\hline 9.1 .4 & GROSSWT1 & Grose woight of sample bottio and contents & grams & 309.843 \\
\hline 9.1 .7 & TAREWTO1 & Tere wolght of PTFE drying/digesting vessel & orame & 168.187 \\
\hline 9.2 .5 & TAREWTO2 & Tare weight of empty dried sample bottie & grams & 33.6309 \\
\hline 9.2 .8 & REFBTLWT & Woight of bottio refillod to initiel liquid lovel & grame & 308.742 \\
\hline 9.2 .10 & DRYWT-01 & Grose weight of drying voseol, ist drying & grame & 168.194 \\
\hline 9.2 .12 & DAYWT-02 & Groes wolght of drying weecel, 2nd drying & grams & 168.195 \\
\hline 9.2.13.1 & AVENETWT & Average of net woight of dried studge & grems & 0.0075 \\
\hline 9.2 .14 & DRYWT-03 & Gross woight of drying vossel, 3rd drying & arame & $n / m$ \\
\hline 9.2 .15 & NETWT-01 & Not weight of dried sludge & oreme & 0.0075 \\
\hline 9.2 .16 .2 & TAREWTO3 & Tare welight of bottle for aludge etorege & groms & 0.0000 \\
\hline 9.2.16.4 & OROSSWT2 & Grose woight of exceee dry sludge + atorage container & grams & 0.0000 \\
\hline 9.2 .16 .5 & NETWT-02 & Net woight of excest dried sludge & grams & 0.0000 \\
\hline 9.2.16.6 & GROSSWT3 & Grose woight of remaining dried sludge to be digented plus drying vessel & grams & 168.195 \\
\hline 9.2.16.7 & NETWT-03 & Net woight of remaining driod sludgo to be digested & orame & 0.0076 \\
\hline 9.2.17 & NETWT-04 & Not weight of dried shudge to be digestod & orame & 0.0075 \\
\hline 8.2.31.3 & VOLDIGO1 & Volume of diluted digentate (oame as volume of volumotric fiask) & $\mathbf{m L}$ & 100 \\
\hline 9.2.31.5 & KOIO-01 & Digestion Fector & & 0.3612 \\
\hline 9.2 .35 & GROSSWT4 & Grose woight of dry digosting veseal plue residus & grams & 188.195 \\
\hline 8.2.36 & APPEARO2 & Appearence of driod residue & $n / a$ & Diseolvod \\
\hline 9.2 .37 & CHILD-01 & LABCOAE number of digestete & $n / \bullet$ & $n / \mathbf{a}$ \\
\hline
\end{tabular}


Table 3. Determination of Data Correction Digestion/Dilution Factors

$K$ Basin Sand Filter Backwash Line

Campaign 20

\begin{tabular}{|c|c|c|c|c|c|c|c|c|}
\hline Column 1 & Column 2 & Column 3 & Column 4 & Column 5 & Column 6 & Column 7 & Column 8 & Column 9* \\
\hline $\begin{array}{c}\text { Customer } \\
\text { Sample Number }\end{array}$ & $\begin{array}{l}\text { Labcore Parent } \\
\text { Sample Number }\end{array}$ & $\begin{array}{c}\text { Sample Bottle } \\
\text { Gross Weight } \\
\text { grams }\end{array}$ & $\begin{array}{l}\text { Bottle Tare } \\
\text { Weight } \\
\text { grams }\end{array}$ & $\begin{array}{l}\text { Density } \\
\text { Factor }\end{array}$ & $\begin{array}{c}\text { Initial Sample Volume } \\
\text { (converted by density) } \\
\text { milliliters }\end{array}$ & $\begin{array}{l}\text { Weight of } \\
\text { Digested Sludge } \\
\text { grams }\end{array}$ & $\begin{array}{l}\text { Volume of Digestate } \\
\text { (Vol flask volume) } \\
\text { milliliters }\end{array}$ & $\begin{array}{l}\text { Digestion Factor } \\
\text { Volume }(\mathrm{mL}) \text { and } \\
\text { weight }(\mathrm{g})\end{array}$ \\
\hline 112KWBMF & S99K0000019 & 309.843 & 33.5309 & 0.998 & 276.866 & 0.0075 & 100.0 & $3.612 \mathrm{E}-01$ \\
\hline
\end{tabular}

Column 3. Derived from step 8.2.8 of LA-505-162, Rev. C-0.

Column 4. Derived from step 8.2.5 of LA-505-162, Rev. C-0.

Column 5. Density of reagent grade water at room temperature.

Column 6. Calculation: (column 3 - column 4 ) $\div$ column 5 .

Column 7. Derived from step 8.2.17 of LA-505-162, Rev. C-0.

Column 8. Derived from step 8.2.31.3 of LA-505-162, Rev. C-0.

Column 9. Calculation: (column 8/ column 6). Digestion factor to correct to total volume and weight fo solids in original sample.

Note: Since all solids were digested, Column 7 is both the weight of solids digested and the original weight of the dried solids. Thus, the ratio is 1 . 
Table 4A. Analytical Results for Digestates

$K$ Basin Sand Filter Backwash Line

Campaign 20

Digestate Results Not Corrected for Digestion Factors or Dilution Factors

\section{Pu-238 by TRU-SPEC Resin}

LA-953-104

\begin{tabular}{|c|c|c|c|c|c|c|c|c|c|c|c|}
\hline \multicolumn{3}{|c|}{ SAMPLE IDENTIFICATION } & \multicolumn{3}{|c|}{ SAMPLE RESULTS } & \multicolumn{6}{|c|}{ QUALITY CONTROL DATA } \\
\hline $\begin{array}{l}\text { Customer } \\
\text { Sample } \\
\text { Number }\end{array}$ & $\begin{array}{l}\text { Laboratory } \\
\text { Sample } \\
\text { Number }\end{array}$ & $\begin{array}{c}\text { Campaign } \\
\text { Number }\end{array}$ & $\begin{array}{l}\text { Sample } \\
\text { Result } \\
\text { UCi/L }\end{array}$ & $\begin{array}{c}\text { Duplicate } \\
\text { Result } \\
\text { uCi/L }\end{array}$ & $\begin{array}{l}\text { Mean } \\
\text { Result } \\
\text { uCi/L }\end{array}$ & $\begin{array}{l}\text { Standard } \\
\% \text { Recoven }\end{array}$ & \begin{tabular}{|c|} 
Preparation \\
Blank \\
UCi/L
\end{tabular} & $\begin{array}{c}\text { Precision } \\
\text { of Duplicate } \\
\text { RPD }\end{array}$ & $\begin{array}{c}\text { Accuracy } \\
\text { of Spike } \\
\% \text { Recovery }\end{array}$ & $\begin{array}{c}\text { Detection } \\
\text { Limit } \\
u C i / L\end{array}$ & \begin{tabular}{|c|} 
Counting \\
Error, \\
Relative \%
\end{tabular} \\
\hline 112KWBMF & S99K000019 & 20 & $<3.83 \mathrm{E}-03$ & $<3.71 E-03$ & $<3.77 E-03$ & $\bar{N} / \mathrm{A}$ & $<3.37 \mathrm{E}-03$ & $\overline{N / A}$ & N/A & $3.37 E-03$ & $\overline{7.8}$ \\
\hline
\end{tabular}


Table 4B. Analytical Results for Digestates

K Basin Sand Filter Backwash Line

Campaign 20

Digestate Results Not Corrected for Digestion Factors or Dilution Factors

Pu-239/240 by TRU-SPEC Resin

LA-953-104

\begin{tabular}{|c|c|c|c|c|c|c|c|c|c|c|c|}
\hline \multicolumn{3}{|c|}{ SAMPLE IDENTIFICATION } & \multicolumn{3}{|c|}{ SAMPLE RESULTS } & \multicolumn{6}{|c|}{ QUALITY CONTROL DATA } \\
\hline $\begin{array}{l}\text { Customer } \\
\text { Sample } \\
\text { Number }\end{array}$ & $\begin{array}{c}\text { Laboratory } \\
\text { Sample } \\
\text { Number }\end{array}$ & $\begin{array}{c}\text { Campaign } \\
\text { Number }\end{array}$ & $\begin{array}{c}\text { Sample } \\
\text { Result } \\
\text { uCi/L }\end{array}$ & $\begin{array}{c}\text { Duplicate } \\
\text { Result } \\
\text { uCi/L }\end{array}$ & $\begin{array}{l}\text { Mean } \\
\text { Result } \\
\mathrm{uCi} / \mathrm{L}\end{array}$ & $\begin{array}{l}\text { Standard } \\
\% \text { Recovery }\end{array}$ & $\begin{array}{c}\text { Preparation } \\
\text { Blank } \\
\mathrm{UCi} / \mathrm{L}\end{array}$ & $\begin{array}{l}\text { Precision } \\
\text { of Duplicate } \\
\text { RPD }\end{array}$ & $\begin{array}{c}\text { Accuracy } \\
\text { of Spike } \\
\% \text { Recovery }\end{array}$ & $\begin{array}{c}\text { Detection } \\
\text { Limit } \\
\text { uCi/L }\end{array}$ & \begin{tabular}{|c|} 
Counting \\
Error, \\
Relative \%
\end{tabular} \\
\hline 112KWBMF & S99K000019 & 20 & $8.07 \mathrm{E}-03$ & $8.34 \mathrm{E}-03$ & $8.21 \mathrm{E}-03$ & 100.8 & $<3.37 \mathrm{E}-03$ & 3.3 & 94.5 & $3.83 \mathrm{E}-03$ & 4.9 \\
\hline
\end{tabular}


Table 4C. Analytical Results for Digestates

$K$ Basin Sand Filter Backwash Line

Campaign 20

Digestate Results Not Corrected for Digestion Factors or Dilution Factors

\section{Uranium by Phosphorescence}

LA-925-009

\begin{tabular}{|c|c|c|c|c|c|c|c|c|c|c|c|}
\hline \multicolumn{3}{|c|}{ SAMPLE IDENTIFICATION } & \multicolumn{3}{|c|}{ SAMPLE RESULTS } & \multicolumn{6}{|c|}{ QUALITY CONTROL DATA } \\
\hline $\begin{array}{l}\text { Customer } \\
\text { Sample } \\
\text { Number }\end{array}$ & $\begin{array}{l}\text { Laboratory } \\
\text { Sample } \\
\text { Number }\end{array}$ & $\begin{array}{c}\text { Campaign } \\
\text { Number }\end{array}$ & $\begin{array}{c}\text { Sample } \\
\text { Result } \\
\text { ug/L }\end{array}$ & $\begin{array}{c}\text { Duplicate } \\
\text { Result } \\
\text { ug/L }\end{array}$ & $\begin{array}{l}\text { Mean } \\
\text { Result } \\
\text { ug/L }\end{array}$ & $\begin{array}{l}\text { Standard } \\
\text { \% Recovery }\end{array}$ & $\begin{array}{c}\text { Preparation } \\
\text { Blank } \\
\text { ug/L }\end{array}$ & $\begin{array}{l}\text { Precision } \\
\text { of Duplicate } \\
\text { RPD }\end{array}$ & $\begin{array}{c}\text { Accuracy } \\
\text { of Spike } \\
\% \text { Recovery }\end{array}$ & $\begin{array}{c}\text { Detection } \\
\text { Limit } \\
\text { ug/L }\end{array}$ & \begin{tabular}{|c|} 
Relative \\
Percent \\
Uncertainty
\end{tabular} \\
\hline 112KWBMF & 599K000019 & 20 & $<3.70 \mathrm{E}+00$ & $<3.70 \mathrm{E}+00$ & $<3.70 \mathrm{E}+00$ & 92.6 & $<3.70 \mathrm{E}+0$ & $\bar{N} / \mathrm{A}$ & $\overline{98.4}$ & $3 . \overline{70 E+00}$ & N/A \\
\hline
\end{tabular}


Table 5. Analytical Results Corrected to Original Sample Volume: Pu-238 by TRU-SPEC Resin

$K$ Basin Sand Fllter Backwash Line

Campaign 20

Data an Corrected for Diaestion Factors and Dilution Factors

$\boldsymbol{\infty}$

PU-238 by TRU-SPEC Resin

LA-953-104

\begin{tabular}{|c|c|c|c|c|c|c|c|c|c|c|c|c|c|c|c|c|}
\hline \multicolumn{3}{|c|}{ SAMPLE IDENTIFICATION } & \multicolumn{7}{|c|}{ SAMPLE RESULTS } & \multicolumn{7}{|c|}{ QUALTY CONTROL DATA } \\
\hline $\begin{array}{l}\text { Customer } \\
\text { Sample } \\
\text { Number }\end{array}$ & $\begin{array}{l}\text { Laboratory } \\
\text { Sample } \\
\text { Number }\end{array}$ & $\begin{array}{l}\text { Campaign } \\
\text { Number }\end{array}$ & $\begin{array}{l}\text { Conversion } \\
\text { Factor } \\
\text { to } \mathrm{uCV} / \mathrm{mL}\end{array}$ & $\begin{array}{c}\text { Digestate } \\
\text { Sample } \\
\text { Result } \\
\text { uCin }\end{array}$ & $\begin{array}{l}\text { Final } \\
\text { Sample } \\
\text { Result } \\
\text { LCU/mL }\end{array}$ & $\begin{array}{l}\text { Digestate } \\
\text { Duplicate } \\
\text { Result } \\
\text { UCIL }\end{array}$ & $\begin{array}{c}\text { Final } \\
\text { Duplicate } \\
\text { Result } \\
\text { uCl/mL }\end{array}$ & $\begin{array}{l}\text { Digestate } \\
\text { Mean } \\
\text { Result } \\
\text { UCVL }\end{array}$ & $\begin{array}{l}\text { Final } \\
\text { Mean } \\
\text { Result } \\
\text { uCi/mL }\end{array}$ & \begin{tabular}{|c|} 
Digestate \\
Preparation \\
Blank \\
UCil \\
\end{tabular} & \begin{tabular}{|c|} 
Final \\
Preparation \\
Blank \\
$\mathbf{u} \mathrm{Cl} / \mathrm{mL}$ \\
\end{tabular} & $\begin{array}{c}\text { Precision } \\
\text { of Duplicate } \\
\text { RPD }\end{array}$ & $\begin{array}{l}\text { Digestate } \\
\text { Detection } \\
\text { Limlt } \\
\text { uCll }\end{array}$ & $\begin{array}{c}\text { Final } \\
\text { Detection } \\
\text { Limit } \\
\text { uCi/mL }\end{array}$ & \begin{tabular}{|c|} 
Estimated \\
Quantitation \\
Limit \\
uCi/mL
\end{tabular} & $\begin{array}{l}\text { Relative } \\
\text { Percent } \\
\text { Counting } \\
\text { Error }\end{array}$ \\
\hline 112KWBMF & S99K000019 & 20 & 3.61E-01 & $<3.83 E-03$ & $<1.38 \mathrm{E}-06$ & $<3.71 E-03$ & $<1.34 E-06$ & $<.77 E-03$ & $<1.36 \mathrm{E}-06$ & $<3.37 \mathrm{E}-03$ & $<3.37 \mathrm{E}-06$ & N/A & $3.37 \mathrm{E}-03$ & $1.22 \mathrm{E}-06$ & $1.22 \mathrm{E}-05$ & 7.8 \\
\hline
\end{tabular}

Notes: The detection limit and retattve percent counting error are besed on the sample results.

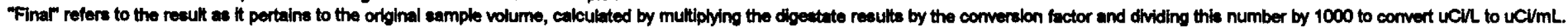


Table 6. Analytical Results Corrected to Original Sample Volume: PU-239/240 by TRU-SPEC Resin K Basin Sand Filter Backwash Line

Campalgn 20

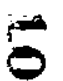
Data are Corrected for Digestion Factors and Dilution Factors

Pu-239/240 by TRU-SPEC Resin

LA-953-104

\begin{tabular}{|c|c|c|c|c|c|c|c|c|c|c|c|c|c|c|c|c|c|c|}
\hline \multicolumn{3}{|c|}{ SAMPLE IDENTIFICATION } & \multicolumn{7}{|c|}{ SAMPLE RESULTS } & \multicolumn{9}{|c|}{ QUALITY CONTROL DATA } \\
\hline $\begin{array}{l}\text { Customer } \\
\text { Sample } \\
\text { Number }\end{array}$ & $\begin{array}{l}\text { Laboratory } \\
\text { Sample } \\
\text { Number }\end{array}$ & $\begin{array}{c}\text { Campaign } \\
\text { Number }\end{array}$ & $\begin{array}{c}\text { Conversion } \\
\text { Factor } \\
\text { to uCi/mL }\end{array}$ & $\begin{array}{c}\text { Digestate } \\
\text { Sample } \\
\text { Result } \\
\text { uCill }\end{array}$ & $\begin{array}{c}\text { Final } \\
\text { Sample } \\
\text { Result } \\
\text { uCi/mL }\end{array}$ & $\begin{array}{c}\text { Digestate } \\
\text { Duplicate } \\
\text { Result } \\
\text { uCi/L }\end{array}$ & $\begin{array}{c}\text { Final } \\
\text { Duplicate } \\
\text { Result } \\
\text { uCi/mL }\end{array}$ & $\begin{array}{c}\text { Digestate } \\
\text { Mean } \\
\text { Result } \\
\text { uCi/L }\end{array}$ & $\begin{array}{c}\text { Final } \\
\text { Mean } \\
\text { Result } \\
\omega \mathrm{Ci} / \mathrm{mL}\end{array}$ & \begin{tabular}{|c|} 
Standard \\
$\%$ Recovery
\end{tabular} & \begin{tabular}{|c|} 
Digestate \\
Preparation \\
Blank \\
uCi/L
\end{tabular} & \begin{tabular}{|c|} 
Final \\
Preparation \\
Blank \\
uCi $/ \mathrm{mL}$
\end{tabular} & \begin{tabular}{|c|} 
Precision \\
of Duplicate \\
RPD
\end{tabular} & $\begin{array}{c}\begin{array}{c}\text { Accuracy } \\
\text { of Spike } \\
\text { \% Recovery }\end{array} \\
\end{array}$ & $\begin{array}{c}\text { Digestate } \\
\text { Detection } \\
\text { Limit } \\
\mathrm{uCi} / \mathrm{L}\end{array}$ & $\begin{array}{c}\text { Final } \\
\text { Detection } \\
\text { Limit } \\
\mathrm{uCi} / \mathrm{mL}\end{array}$ & \begin{tabular}{|c|} 
Estimated \\
Quantitation \\
Limit \\
$\mathrm{uCi} / \mathrm{mL}$ \\
\end{tabular} & $\begin{array}{c}\text { Relative } \\
\text { Percent } \\
\text { Counting } \\
\text { Error }\end{array}$ \\
\hline 112KWBMF & S99K000019 & 20 & $3.612 \mathrm{E}-01$ & $8.07 \mathrm{E}-03$ & $2.91 \mathrm{E}-06$ & $8.34 \mathrm{E}-03$ & $3.01 E-06$ & $8.21 \mathrm{E}-03$ & $2.96 \mathrm{E}-06$ & 100.8 & $<3.37 \mathrm{E}-03$ & $<3.37 \mathrm{E}-06$ & 3.3 & 94.5 & $3.83 \mathrm{E}-03$ & $1.38 \mathrm{E}-06$ & $1.38 \mathrm{E}-05$ & 4.9 \\
\hline
\end{tabular}

Notes: The detection limit and relative percent counting error are based on the sample results.

"Final" refers to the result as it pertains to the original sample volume, calculated by multiplying the digestate results by the conversion factor and dividing this number by 1000 to convert uCi/ to uCi/mL. 
$\because$

Table 7. Analytical Results Corrected to Original Sample Volume: Uranium by Phosphorescence K Basin Sand Filter Backwash Line

$$
\text { Campaign } 20
$$

Data are Corrected for Digestion Factors and Dilution Factors

Uranium by Phosphorescence

LA-925-009

\begin{tabular}{|c|c|c|c|c|c|c|c|c|c|c|c|c|c|c|c|c|c|c|}
\hline \multicolumn{3}{|c|}{ SAMPLE IDENTIFICATION } & \multicolumn{7}{|c|}{ SAMPLE RESULTS } & \multicolumn{9}{|c|}{ QUALITY CONTROL DATA } \\
\hline $\begin{array}{l}\text { Customer } \\
\text { Sample } \\
\text { Number }\end{array}$ & $\begin{array}{l}\text { Laboratory } \\
\text { Sample } \\
\text { Number }\end{array}$ & $\begin{array}{c}\text { Campaign } \\
\text { Number }\end{array}$ & \begin{tabular}{|c|} 
Conversion \\
Factor
\end{tabular} & $\begin{array}{c}\text { Digestate } \\
\text { Sample } \\
\text { Result } \\
\text { ug } /\end{array}$ & $\begin{array}{c}\text { Finai } \\
\text { Sample } \\
\text { Result } \\
u g / m L\end{array}$ & $\begin{array}{c}\text { Digestate } \\
\text { Duplicate } \\
\text { Result } \\
\text { ug/L }\end{array}$ & $\begin{array}{c}\text { Final } \\
\text { Duplicate } \\
\text { Result } \\
\text { ug/mL }\end{array}$ & $\begin{array}{c}\text { Digestate } \\
\text { Mean } \\
\text { Resuit } \\
\text { ug/L }\end{array}$ & $\begin{array}{l}\text { Final } \\
\text { Mean } \\
\text { Result } \\
\mathrm{ug} / \mathrm{mL}\end{array}$ & $\begin{array}{l}\text { Standard } \\
\% \text { Recovery }\end{array}$ & \begin{tabular}{|c|} 
Digestate \\
Preparation \\
Blank \\
ug $/ \mathrm{L}$
\end{tabular} & $\begin{array}{c}\text { Final } \\
\text { Preparation } \\
\text { Blank } \\
\text { ug/mL }\end{array}$ & $\begin{array}{c}\begin{array}{c}\text { Precision } \\
\text { of Duplicate } \\
\text { RPD }\end{array} \\
\end{array}$ & $\begin{array}{c}\begin{array}{c}\text { Accuracy } \\
\text { of Spike } \\
\% \text { Recovery }\end{array} \\
\text { \% }\end{array}$ & $\begin{array}{l}\text { Digestate } \\
\text { Detection } \\
\text { Limit } \\
\text { ug/L }\end{array}$ & $\begin{array}{c}\text { Final } \\
\text { Detection } \\
\text { Limit } \\
\text { ug } / \mathrm{mL} .\end{array}$ & \begin{tabular}{c|} 
Estimated \\
Quantitation \\
Limit \\
$\mathrm{uCi} / \mathrm{mL}$ \\
\end{tabular} & \begin{tabular}{|c|} 
Relative \\
Percent \\
Uncertainty
\end{tabular} \\
\hline $112 \mathrm{KWBMF}$ & S99K000019 & $\overline{20}$ & 3.61E-01 & $<3.70 \mathrm{E}+00$ & $<1.34 \mathrm{E}-03$ & $<3.70 \mathrm{E}+00$ & $<1.34 E-03$ & $<3.70 \mathrm{E}+00$ & $<1.34 E-03$ & $\overline{92.6}$ & $<3.70 E+00$ & $<3.70 \mathrm{E}-03$ & $N / A$ & 98.4 & $3.70 \mathrm{E}+00$ & $1.34 \mathrm{E}-03$ & $1.34 \mathrm{E}-02$ & N/A \\
\hline
\end{tabular}

Notes: The detection limit and relative percent uncertainty are based on the sample results.

"Final" refers to the result as it pertains to the original sample volume, calculated by multiplying the digestate results by the conversion factor and dividing by 1000 to convert uCin to uCi/mL. 
:

1

HNF-1673 REV. 0

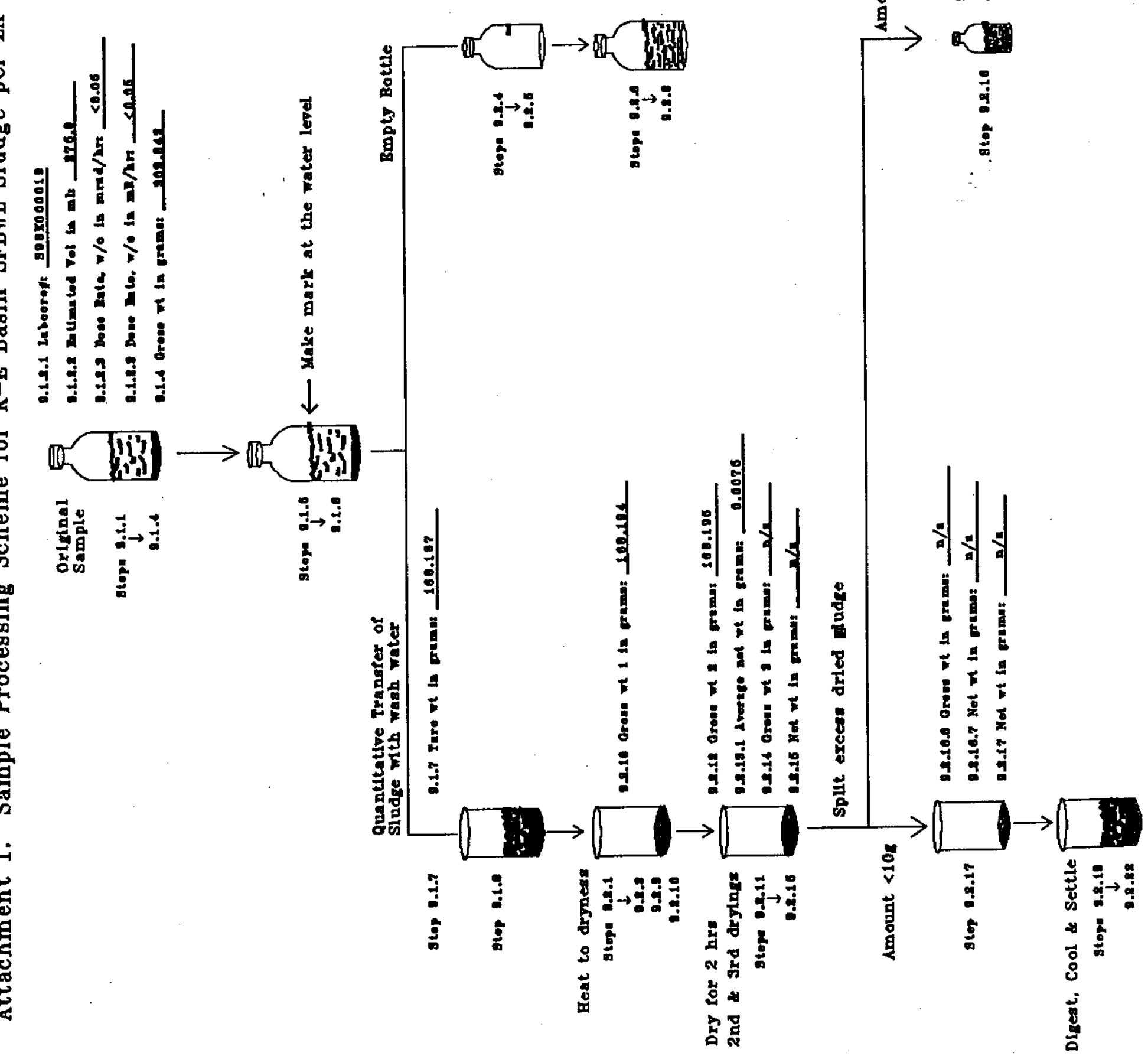


HNF-1673 REV. 0
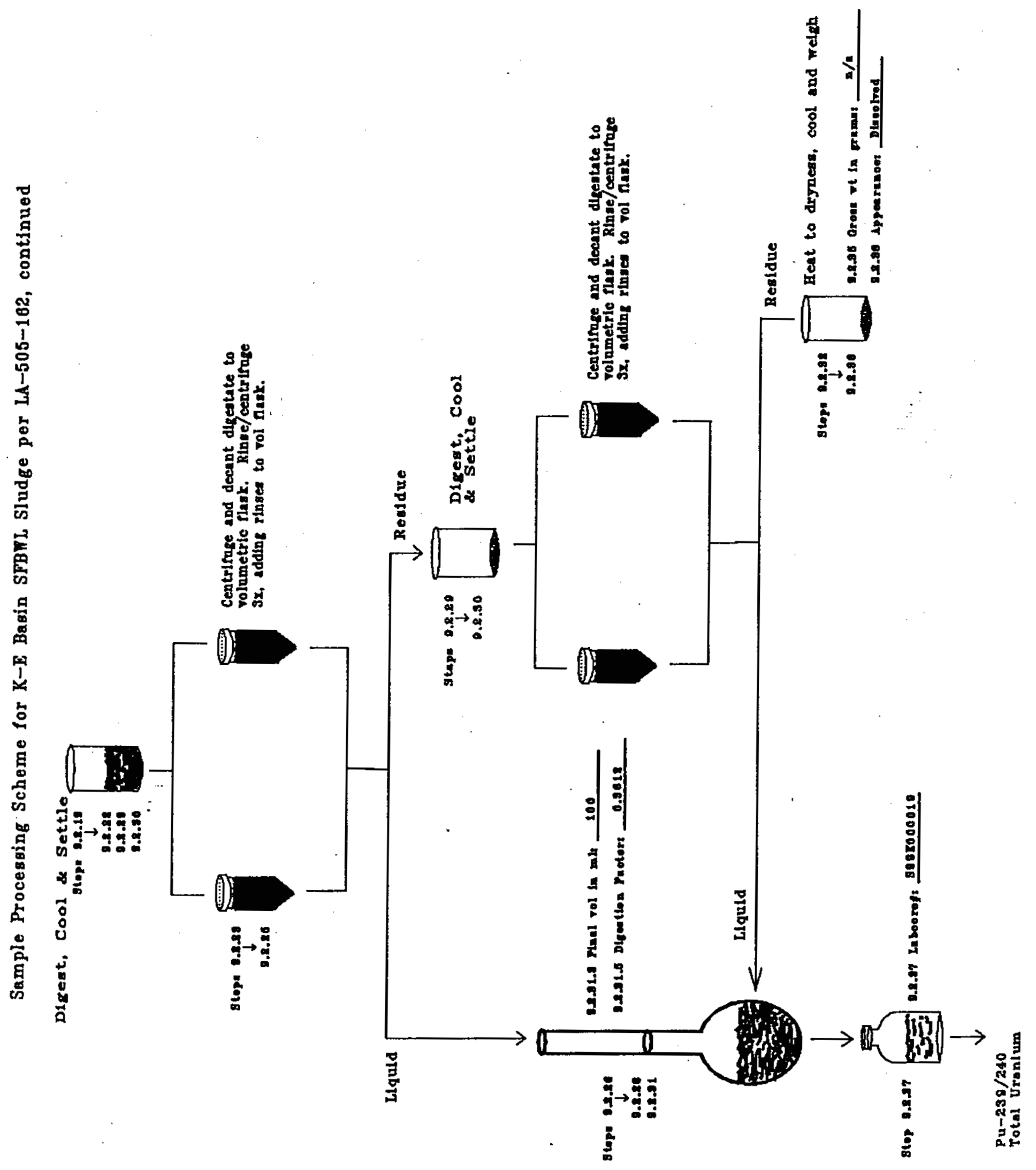


\section{DISTRIBUTION SHEET}

\begin{tabular}{|c|c|c|c|c|}
\hline \multirow{2}{*}{$\begin{array}{l}\text { To } \\
\text { Distribution }\end{array}$} & \multirow{2}{*}{\multicolumn{2}{|c|}{ From }} & \multicolumn{2}{|c|}{ Page 1 of 1} \\
\hline & & & Date: & $06 / 28 / 99$ \\
\hline \multirow{2}{*}{$\begin{array}{l}\text { Project Title/Wor } \\
\text { HNF-1673 Rev. O } \\
\text { Characterization }\end{array}$} & \multirow{2}{*}{\multicolumn{2}{|c|}{$\begin{array}{l}\text { Sandfilter Backwash Line } \\
\text { Analytical Results for Campaign 20" }\end{array}$}} & \multicolumn{2}{|c|}{ EDT NO.: EDT-611474 } \\
\hline & & & \multicolumn{2}{|c|}{ ECN NO.: N/A } \\
\hline & Name & $\begin{array}{l}\text { Text With } \\
\text { all Attach }\end{array}$ & $\begin{array}{l}\text { EDT/ECN } \\
\text { ONLY }\end{array}$ & \\
\hline
\end{tabular}

DE\&S Hanford. Inc.
R. B. Baker
D. W. Bergman
S. P. Burke
D. D. Faris-Zyph
C. D. Lucas
D. H. Sandoz

$\begin{array}{ll}40-40 & X \\ \times 3-79 & X \\ X 3-74 & X \\ X 3-56 & X \\ X 3-79 & X \\ X 3-79 & X\end{array}$

Lockheed Martin Hanford Corp.
A. E. Young
R1-10

$X$
$X$
$X$
$X$
$X$
$X$

Lockheed Martin Services. Inc.

Central Files

A3-88 $\quad 1$

Waste Management of Hanford. Inc.
D. C. Hetzer
K. L. Powell
C. M. Seidel
F. H. Steen
L. A. Diaz

S6-31

S3-30

S3-30

T6-12

T6-12

T6-12

$X$
$X$
$X$
$X$
$X$
$X$

* Needs only releasing paperwork, not a copy of the released document. 